\title{
Truncated Prediction Output Feedback Control of a Class of Lipschitz Nonlinear Systems with Input Delay
}

\author{
Zongyu Zuo, Member, IEEE, Zongli Lin, Fellow, IEEE, and Zhengtao Ding, Senior Member, IEEE
}

\begin{abstract}
This brief addresses an output feedback control design for Lipschitz nonlinear systems in the presence of input delay. A nonlinear observer is introduced to estimate the system states. A truncated predictor state feedback, which is the conventional predictor state feedback law with the distributed term dropped, is implemented with the estimated states. LyapunovKrasovskii functionals are constructed to establish conditions under which this observer-based truncated predictor feedback law asymptotically stabilize the system at the origin. The effectiveness of the proposed method is demonstrated on the Chua's circuit.
\end{abstract}

Index Terms-Chua's circuit, input delay, Lipschitz nonlinearity, output feedback

\section{INTRODUCTION}

Time delays are ubiquitous in practical systems due to the time taken for transmission of signals, transport of materials, etc. The presence of time delays, if not considered in the controller design, may seriously degrade the performance of the controlled system. For the analysis and control of circuit systems, time delay must be included during the process of modeling when the geometric dimensions become electrically large and the frequency content of signal waveform increases [1]. Indeed, the importance of addressing delay in the control design has been well recognized for a long time [2].

One basic idea in tackling input delay is to predict the evolution of the state variable for the delay period and then use the predicted state for control. One of the early results for input-delayed linear systems is the Smith predictor [3], which is a frequency-domain method, and has been widely used in industry. In the time domain, it has been shown that a state predictor satisfies a delay-free state space equation, and control design can be developed with the prediction [4]. The predictor-based method transforms the state with an integral operator in the state transformation. However, it encounters difficulties in practical implementation and does not guarantee exponential stability of the original system [5], [6], despite the control design is simplified by the state transformation. An alternative method, based on the prediction and referred to

This work was supported by the National Natural Science Foundation of China (Nos. 61203022 and 61273105), the US National Science Foundation (CMMI-1462171), and the China Scholarship Council (CSC).

Z. Zuo is with the Seventh Research Division, and Science and Technology on Aircraft Control Laboratory, Beihang University (BUAA), Beijing 100191, China (zzybobby@buaa.edu.cn)

Z. Lin is with Charles L., Brown Department of Electrical and Computer Engineering, University of Virginia, Charlottesville, VA 22904-4743, USA (zl5y@virginia.edu).

Z. Ding is with Control Systems Center, School of Electrical and Electronic Engineering, University of Manchester, Sackville Street Building, Manchester M13 9PL, UK (zhengtao.ding@ manchester.ac.uk, the corresponding author)

Copyright (c) 2015 IEEE. Personal use of this material is permitted. However, permission to use this material for any other purposes must be obtained from the IEEE by sending an email to pubs-permissions@ieee.org. as the Truncated Prediction Feedback (TPF), is to ignore the troublesome integral part and make the prediction based solely on the exponential of the system matrix. This idea stemmed [7], where the input is kept low such that its contribution to the prediction can be safely neglected [8]-[10], where both state feedback and output feedback designs are considered. The TPF developed in [8]-[10] are for open loop systems that are not exponentially unstable. The TPF for general open loop systems, including exponentially unstable ones, was later developed in [11].

Control design for nonlinear systems with input delay is much more involved than that for the linear systems counterpart. Several results can be found in the literature [12]-[17] that address this problem. However, few results are available on output feedback control for input-delayed nonlinear systems. Although there have been many results developed on the observer design for Lipschitz nonlinear systems [18]-[20], the output feedback or observer-based control design is nontrivial, due to the fact that the separation principle does not hold in general for nonlinear systems.

This brief considers the output feedback control design for a class of Lipschitz nonlinear systems with input delay. The state feedback controller is not applicable in many practical occasions where only system output signals are accessible. An estimated state based truncated predictor feedback control is developed, which leads to several extra integral terms corresponding to the system state and the observation error, all resulting from the truncation errors. To guarantee the closedloop stability, a set of conditions for the output feedback control problem are established. Different from the stability analysis given in [21] for linear systems, the stability analysis in this brief is put in the framework of Lyapunov-Krasovskii functionals. To facilitate the calculation, we convert these conditions to LMIs with a set of iterative scalar parameters. Although the controller and the observer cannot be designed independently, the observer gain can be calculated for a fixed solution to the conditions for the control gain design which can be solved first. Stabilization of a chaotic circuit is introduced as an example to demonstrate the proposed design.

\section{Problem Statement}

We consider control design for a class of Lipschitz nonlinear systems with input delay

$$
\begin{aligned}
\dot{x}(t) & =A x(t)+B u(t-h)+\phi(x(t)), \\
y(t) & =C x(t),
\end{aligned}
$$

where $x \in \mathbb{R}^{n}$ is the state, $u \in \mathbb{R}^{p}$ is the input, $y \in \mathbb{R}^{q}$ is the measurement output, $h \in \mathbb{R}_{+}$is the input delay, $A \in$ inger obtained from from low gain control of the systems with input saturation 
$\mathbb{R}^{n \times n}, B \in \mathbb{R}^{n \times p}$ and $C \in \mathbb{R}^{q \times n}$ are constant matrices with $(A, B)$ being controllable and $(A, C)$ being observable, and $\phi: \mathbb{R}^{n} \rightarrow \mathbb{R}^{n}, \phi(0)=0$, is a Lipschitz nonlinear function with a Lipschitz constant $\gamma$. For any two constant vectors $a, b \in \mathbb{R}^{n}$,

$$
\|\phi(a)-\phi(b)\| \leq \gamma\|a-b\| .
$$

From the system dynamics (1), we have

$$
\begin{aligned}
x(t)= & e^{A h} x(t-h)+\int_{t-h}^{t} e^{A(t-\tau)} B u(\tau-h) \mathrm{d} \tau \\
& +\int_{t-h}^{t} e^{A(t-\tau)} \phi(x(\tau)) \mathrm{d} \tau .
\end{aligned}
$$

The first term, $e^{A h} x(t-h)$, is a truncated predictor of the system state at $t$ based on $x(t-h)$. The truncated predictor output feedback control input is constructed as

$$
\begin{aligned}
\dot{\hat{x}}(t)= & A \hat{x}(t)+B u(t-h)+\phi(\hat{x}(t)) \\
& +L(C \hat{x}(t)-y(t)), \\
u(t)= & K e^{A h} \hat{x}(t),
\end{aligned}
$$

where $K$ and $L$ denote the control gain matrix and the observer gain matrix, respectively, to be designed later. Let $\tilde{x}(t)=\hat{x}(t)-x(t)$. Substituting (6) into (1) and using (4) and (5) give the resultant closed-loop dynamics as

$$
\begin{aligned}
\dot{x}(t)= & A x(t)+B K e^{A h}(x(t-h)+\tilde{x}(t-h))+\phi(x(t)) \\
= & (A+B K) x(t)-B K\left(\lambda_{1}(t)+\lambda_{2}(t)+\lambda_{3}(t)\right) \\
& +B K e^{A h} \tilde{x}(t-h)+\phi(x(t)) \\
\dot{\tilde{x}}(t)= & \dot{\hat{x}}(t)-\dot{x}(t) \\
= & (A+L C) \tilde{x}(t)+\phi(\hat{x}(t))-\phi(x(t))
\end{aligned}
$$

where

$$
\begin{aligned}
& \lambda_{1}(t)=\int_{t-h}^{t} e^{A(t-\tau)} B K e^{A h} x(\tau-h) \mathrm{d} \tau, \\
& \lambda_{2}(t)=\int_{t-h}^{t} e^{A(t-\tau)} B K e^{A h} \tilde{x}(\tau-h) \mathrm{d} \tau, \\
& \left.\lambda_{3}(t)=\int_{t-h}^{t} e^{A(t-\tau)} \phi(x(\tau))\right) \mathrm{d} \tau .
\end{aligned}
$$

It is worth mentioning that the extra terms $\lambda_{2}$ and $\lambda_{3}$ in (7) are due to the errors in the truncation, which correspond to the observation error and the nonlinear term $\phi(x(t))$, respectively.

Specifically, the controller and the observer gains in (5) and (6) are defined, respectively, as

$$
K=-B^{\mathrm{T}} P_{1} \quad \text { and } \quad L=-P_{2}^{-1} C^{\mathrm{T}},
$$

where $P_{1}$ and $P_{2}$ are both positive definite matrices to be designed.

The control objective in this brief is to design the output feedback control input (5) and (6) such that the closed-loop system (7) is globally asymptotically stable at the origin. In other words, the control design problem is to find possible positive definite matrices $P_{1}$ and $P_{2}$ such that the control law (5) and (6) with the gains (12) globally stabilizes system (1).

\section{Preliminary Results}

This section presents a couple of preliminary results, which are useful for the stability analysis.

Lemma 1: [22] For a positive definite matrix $P$, and a function $x:[a, b] \rightarrow \mathbb{R}^{n}$, with $a, b \in \mathbb{R}$ and $b>a$, the following inequality holds

$$
\begin{aligned}
\left(\int_{a}^{b} x^{\mathrm{T}}(\tau) \mathrm{d} \tau\right) P & \left(\int_{a}^{b} x(\tau) \mathrm{d} \tau\right) \\
& \leq(b-a) \int_{a}^{b} x^{\mathrm{T}}(\tau) P x(\tau) \mathrm{d} \tau .
\end{aligned}
$$

Lemma 2: [17] For a positive definite matrix $P$, the following identity holds

$$
e^{A^{\mathrm{T}} t} P e^{A t}-e^{\omega t} P=-e^{\omega t} \int_{0}^{t} e^{-\omega \tau} e^{A^{\mathrm{T}} \tau} R e^{A \tau} \mathrm{d} \tau,
$$

where $\omega \geq 0$ is a scalar and

$$
R=-A^{\mathrm{T}} P-P A+\omega P .
$$

Furthermore, if $R$ is positive definite, then

$$
e^{A^{\mathrm{T}} t} P e^{A t}<e^{\omega t} P \text {. }
$$

\section{MAIN RESUlt}

This section establishes the conditions for the positive definite matrices $P_{1}$ and $P_{2}$ such that the truncated predictor output feedback control in (5) and (6) stabilizes the system (1).

To start the analysis, let us try a Lyapunov function candidate

$$
V_{0}=x^{\mathrm{T}}(t) P_{1} x(t)+\tilde{x}^{\mathrm{T}}(t) P_{2} \tilde{x}(t) .
$$

With (12), the closed-loop dynamics in (7) and (8) can be re-written as

$$
\begin{aligned}
\dot{x}(t)= & \left(A-B B^{\mathrm{T}} P_{1}\right) x(t)+B B^{\mathrm{T}} P_{1}\left(\lambda_{1}(t)+\lambda_{2}(t)+\lambda_{3}(t)\right) \\
& -B B^{\mathrm{T}} P_{1} e^{A h} \tilde{x}(t-h)+\phi(x(t)), \\
\dot{\tilde{x}}(t)= & \left(A-P_{2}^{-1} C^{\mathrm{T}} C\right) \tilde{x}(t)+\phi(\hat{x}(t))-\phi(x(t)) .
\end{aligned}
$$

The derivative of $V_{0}$ along the trajectories of (17) and (18) can be evaluated as

$$
\begin{aligned}
\dot{V}_{0}= & x^{\mathrm{T}}(t)\left(A^{\mathrm{T}} P_{1}+P_{1} A-2 P_{1} B B^{\mathrm{T}} P_{1}\right) x(t) \\
& +\tilde{x}^{\mathrm{T}}(t)\left(A^{\mathrm{T}} P_{2}+P_{2} A-2 C^{\mathrm{T}} C\right) \tilde{x}(t) \\
& +2 x^{\mathrm{T}}(t) P_{1} B B^{\mathrm{T}} P_{1}\left(\lambda_{1}(t)+\lambda_{2}(t)+\lambda_{3}(t)\right) \\
& -2 x^{\mathrm{T}}(t) P_{1} B B^{\mathrm{T}} P_{1} e^{A h} \tilde{x}(t-h)+2 x^{\mathrm{T}}(t) P_{1} \phi(x) \\
& +2 \tilde{x}(t)^{\mathrm{T}} P_{2}(\phi(\hat{x})-\phi(x)) \\
\leq & x^{\mathrm{T}}(t)\left(A^{\mathrm{T}} P_{1}+P_{1} A-P_{1} B B^{\mathrm{T}} P_{1}+P_{1} P_{1}\right. \\
& \left.+3\left(P_{1} B B^{\mathrm{T}} P_{1}\right)^{2}\right) x(t)+\Delta_{1}(t)+\Delta_{2}(t)+\Delta_{3}(t) \\
& +\tilde{x}^{\mathrm{T}}(t)\left(A^{\mathrm{T}} P_{2}+P_{2} A-2 C^{\mathrm{T}} C+P_{2} P_{2}\right) \tilde{x}(t) \\
& +\phi^{\mathrm{T}}(x) \phi(x)+(\phi(\hat{x})-\phi(x))^{\mathrm{T}}(\phi(\hat{x})-\phi(x)) \\
& +\tilde{x}^{\mathrm{T}}(t-h) e^{A^{\mathrm{T}} h} P_{1} B B^{\mathrm{T}} P_{1} e^{A h} \tilde{x}(t-h) \\
\leq & x^{\mathrm{T}}(t)\left(A^{\mathrm{T}} P_{1}+P_{1} A-P_{1} B B^{\mathrm{T}} P_{1}+\left(3 \alpha^{2}+1\right) P_{1} P_{1}\right. \\
& \left.+\gamma^{2} I\right) x(t)+\Delta_{1}(t)+\Delta_{2}(t)+\Delta_{3}(t) \\
& +\tilde{x}^{\mathrm{T}}(t)\left(A^{\mathrm{T}} P_{2}+P_{2} A-2 C^{\mathrm{T}} C+P_{2} P_{2}+\gamma^{2} I\right) \tilde{x}(t) \\
& +\alpha e^{\omega_{1} h} \tilde{x}^{\mathrm{T}}(t-h) P_{1} \tilde{x}(t-h),
\end{aligned}
$$


where Lemma 2 is used to derive the last inequality provided that

$$
R_{1}=-A^{\mathrm{T}} P_{1}-A P_{1}+\omega_{1} P_{1}>0
$$

with $\omega_{1} \geq 0, \alpha$ is positive real numbers such that

$$
\alpha I \geq P^{\frac{1}{2}} B B^{\mathrm{T}} P^{\frac{1}{2}}
$$

and

$$
\Delta_{i}(t)=\lambda_{i}^{\mathrm{T}}(t) \lambda_{i}(t), i=1,2,3
$$

The remaining part of the analysis is to explore the bounds on $\Delta_{i}, i=1,2,3$.

From (9) and (10), and by Lemmas 1 and 2 with the condition

$$
R_{2}=-A^{\mathrm{T}}-A+\omega_{2} I>0, \omega_{2} \geq 0,
$$

we have

$$
\begin{aligned}
\Delta_{1}= & \int_{t-h}^{t} x^{\mathrm{T}}(\tau-h) e^{A^{\mathrm{T}} h} P_{1} B B^{\mathrm{T}} e^{A^{\mathrm{T}}(t-\tau)} \mathrm{d} \tau \\
& \left.\times \int_{t-h}^{t} e^{A(t-\tau)} B B^{\mathrm{T}} P_{1} e^{A h} x(\tau-h)\right) \mathrm{d} \tau \\
\leq & h \int_{t-h}^{t} x^{\mathrm{T}}(\tau-h) e^{A^{\mathrm{T}} h} P_{1} B B^{\mathrm{T}} e^{A^{\mathrm{T}}(t-\tau)} \\
& \left.\times e^{A(t-\tau)} B B^{\mathrm{T}} P_{1} e^{A h} x(\tau-h)\right) \mathrm{d} \tau \\
\leq & \alpha^{2} h \int_{t-h}^{t} e^{\omega_{2}(t-\tau)} x^{\mathrm{T}}(\tau-h) e^{A^{\mathrm{T}} h} e^{A h} x(\tau-h) \mathrm{d} \tau \\
\leq & \alpha^{2} h e^{2 \omega_{2} h} \int_{t-h}^{t} x^{\mathrm{T}}(\tau-h) x(\tau-h) \mathrm{d} \tau,
\end{aligned}
$$

and similarly,

$$
\Delta_{2} \leq \alpha^{2} h e^{2 \omega_{2} h} \int_{t-h}^{t} \tilde{x}^{\mathrm{T}}(\tau-h) \tilde{x}(\tau-h) \mathrm{d} \tau
$$

where the inequality (21) is used.

On the other hand, from (3) and (11), we have

$$
\begin{aligned}
\Delta_{3} & =\int_{t-h}^{t} \phi^{\mathrm{T}}(x(\tau)) e^{A^{\mathrm{T}}(t-\tau)} \mathrm{d} \tau \int_{t-h}^{t} e^{A(t-\tau)} \phi(x(\tau)) \mathrm{d} \tau \\
& \leq h \int_{t-h}^{t} \phi^{\mathrm{T}}(x(\tau)) e^{A^{\mathrm{T}}(t-\tau)} e^{A(t-\tau)} \phi(x(\tau)) \mathrm{d} \tau \\
& \leq h \int_{t-h}^{t} e^{\omega_{2}(t-\tau)} \phi^{\mathrm{T}}(x(\tau)) \phi(x(\tau)) \mathrm{d} \tau \\
& \leq h e^{\omega_{2} h} \gamma^{2} \int_{t-h}^{t} x^{\mathrm{T}}(\tau) x(\tau) \mathrm{d} \tau
\end{aligned}
$$

For the term $\Delta_{1}$ shown in (23), we consider the following Krasovskii functional

$$
V_{1}(t)=e^{h} \int_{t-h}^{t}\left(e^{\tau-t} x^{\mathrm{T}}(\tau-h) x(\tau-h)+x^{\mathrm{T}}(\tau) x(\tau)\right) \mathrm{d} \tau .
$$

A direct evaluation gives that

$$
\begin{aligned}
\dot{V}_{1}(t)= & -e^{h} \int_{t-h}^{t} e^{\tau-t} x^{\mathrm{T}}(\tau-h) x(\tau-h) \mathrm{d} \tau \\
& -x^{\mathrm{T}}(t-2 h) P_{1} x(t-2 h)+e^{h} x^{\mathrm{T}}(t) x(t) \\
\leq & -\int_{t-h}^{t} x^{\mathrm{T}}(\tau-h) x(\tau-h) \mathrm{d} \tau+e^{h} x^{\mathrm{T}}(t) x(t)
\end{aligned}
$$

For the term $\Delta_{2}$ shown in (24), we consider the following Krasovskii functional

$V_{2}(t)=e^{h} \int_{t-h}^{t}\left(e^{\tau-t} \tilde{x}^{\mathrm{T}}(\tau-h) \tilde{x}(\tau-h)+\tilde{x}^{\mathrm{T}}(\tau) \tilde{x}(\tau)\right) \mathrm{d} \tau$.

A direct evaluation gives that

$$
\dot{V}_{2}(t) \leq-\int_{t-h}^{t} \tilde{x}^{\mathrm{T}}(\tau-h) \tilde{x}(\tau-h) \mathrm{d} \tau+e^{h} \tilde{x}^{\mathrm{T}}(t) \tilde{x}(t) .
$$

For the term $\Delta_{3}$ shown in (25), we consider the following Krasovskii functional

$$
V_{3}(t)=e^{h} \int_{t-h}^{t} e^{\tau-t} x^{\mathrm{T}}(\tau) x(\tau) \mathrm{d} \tau
$$

A direct evaluation gives that

$$
\begin{aligned}
\dot{V}_{3}(t)= & -e^{h} \int_{t-h}^{t} e^{\tau-t} x^{\mathrm{T}}(\tau) x(\tau) \mathrm{d} \tau \\
& +e^{h} x^{\mathrm{T}}(t) x(t)-x^{\mathrm{T}}(t-h) x(t-h) \\
\leq & -\int_{t-h}^{t} x^{\mathrm{T}}(\tau) x(\tau) \mathrm{d} \tau+e^{h} x^{\mathrm{T}}(t) x(t) .
\end{aligned}
$$

For the term $\tilde{x}^{\mathrm{T}}(t-h) P_{1} \tilde{x}(t-h)$ in (19), we consider the following Krasovskii functional

$$
V_{4}(t)=\int_{t-h}^{t} \tilde{x}^{\mathrm{T}}(\tau) P_{1} \tilde{x}(\tau) \mathrm{d} \tau .
$$

A direct evaluation gives that

$$
\dot{V}_{4}(t)=\tilde{x}^{\mathrm{T}}(t) P_{1} \tilde{x}(t)-\tilde{x}^{\mathrm{T}}(t-h) P_{1} \tilde{x}(t-h) .
$$

Now, let

$$
V=V_{0}+\alpha^{2} h e^{2 \omega_{2} h}\left(V_{1}+V_{2}\right)+h e^{\omega_{2} h} \gamma^{2} V_{3}+\alpha e^{\omega_{1} h} V_{4} .
$$

From (19) and (23)-(29), we have that

$$
\dot{V} \leq x^{\mathrm{T}}(t) Q_{1} x(t)+\tilde{x}^{\mathrm{T}}(t) Q_{2} \tilde{x}(t),
$$

where

$$
\begin{aligned}
Q_{1}= & A^{\mathrm{T}} P_{1}+P_{1} A-P_{1} B B^{\mathrm{T}} P_{1}+\left(3 \alpha^{2}+1\right) P_{1} P_{1} \\
& +\alpha^{2} h e^{\left(2 \omega_{2}+1\right) h} I+\left(h e^{\left(\omega_{2}+1\right) h}+1\right) \gamma^{2} I, \\
Q_{2}= & A^{\mathrm{T}} P_{2}+P_{2} A-2 C^{\mathrm{T}} C+P_{2} P_{2}+\alpha e^{\omega_{1} h} P_{1} \\
& +\left(\gamma^{2}+\alpha^{2} h e^{\left(2 \omega_{2}+1\right) h}\right) I .
\end{aligned}
$$

Theorem 1: Consider the nonlinear system (1). The output feedback control law (5) and (6) with (12) globally asymptotically stabilizes the system (1) at the origin if the following conditions are satisfied, for $W=P_{1}^{-1}>0, P_{2}>0, \alpha>0$, $\omega_{1} \geq 0, \omega_{2} \geq 0$,

$$
\begin{aligned}
& \alpha W \geq B B^{\mathrm{T}}, \\
& W\left(A-\frac{1}{2} \omega_{1} I\right)^{\mathrm{T}}+\left(A-\frac{1}{2} \omega_{1} I\right) W<0, \\
& \left(A-\frac{1}{2} \omega_{2} I\right)^{\mathrm{T}}+\left(A-\frac{1}{2} \omega_{2} I\right)<0, \\
& {\left[\begin{array}{cc}
H_{1}-B B^{\mathrm{T}} & W \\
W & -\frac{1}{\bar{\gamma}} I
\end{array}\right]<0,} \\
& {\left[\begin{array}{cc}
H_{2}-2 C^{\mathrm{T}} C & P_{2} \\
P_{2} & -I
\end{array}\right]<0,}
\end{aligned}
$$


where

$$
\begin{aligned}
\bar{\gamma} & =\alpha^{2} h e^{\left(2 \omega_{2}+1\right) h}+\left(h e^{\left(\omega_{2}+1\right) h}+1\right) \gamma^{2}, \\
H_{1} & =\left(3 \alpha^{2}+1\right) I+W A^{\mathrm{T}}+A W, \\
H_{2} & =\left(\gamma^{2}+\alpha^{2} h e^{\left(2 \omega_{2}+1\right) h}\right) I+A^{\mathrm{T}} P_{2}+P_{2} A+\alpha e^{\omega_{1} h} P_{1} .
\end{aligned}
$$

Proof: It is easy to see that the conditions in (33), (34) and (35) are equivalent to the conditions specified in (21), (20) and (22), respectively. With (31) and (32), it can be shown by Schur Complement that conditions (36) and (37) are respectively equivalent to $Q_{1}<0$ and $Q_{2}<0$, which further implies from (30) that $\dot{V}(t)<0$. Thus, the closedloop dynamics (7) and (8) are globally asymptotically stable at the origin. This completes the proof.

Remark 1: The conditions shown in (33)-(37) can be checked by standard LMI routines for a set of fixed values. Note that $\omega_{2}$ in (35) can be determined independently. The conditions in (33), (34) and (36) have to be checked simultaneously. With the possible solution $P_{1}$ obtained by computing (33), (34) and (36), a possible solution $P_{2}$ of (37) can then be computed for a fixed $P_{1}$, which indicates that the designs of the observer and the feedback law are coupled.

\section{AN EXAMPLE}

We consider an input-delayed voltage feedback Chua's circuit system, as shown in Fig. 1. The new output feedback Chua's circuit [23] with input delay can be described by the following equations

$$
\left\{\begin{array}{l}
\frac{\mathrm{d} v_{1}}{\mathrm{~d} t}(t)=\frac{G}{C_{1}}\left(v_{2}(t)-v_{1}(t)\right)-\frac{1}{C_{1}} f\left(v_{1}\right), \\
\frac{\mathrm{d} v_{2}}{\mathrm{~d} t}(t)=\frac{1}{C_{3}} i_{3}(t)-\frac{G}{C_{2}}\left(v_{2}(t)-v_{1}(t)\right), \\
\frac{\mathrm{d} i_{3}}{\mathrm{~d} t}(t)=-\frac{1}{L}\left(v_{2}(t)+R_{0} i_{3}(t)+u(t-h)\right),
\end{array}\right.
$$

where $v_{1}$ and $v_{2}$ denote the voltage across $C_{1}$ and $C_{2}$, respectively, $i_{3}$ denotes the current through $L, G=1 / R$, and $f\left(v_{1}\right)$ characterizes the $v-i$ property of the nonlinear resistor $N_{R}$ with a slope $G_{a}$ in the inner region and $G_{b}$ in the outer region, and the breakpoint voltage $E$ of the Chua's diode, i.e., $f\left(v_{1}\right)=G_{b} v_{1}+0.5\left(G_{a}-G_{b}\right)\left(\left|v_{1}+E\right|-\left|v_{1}-E\right|\right)$.

Let $x_{1}=v_{1} / E, x_{2}=v_{2} / E, x_{3}=i_{3} /(E G), \varepsilon=C_{2} / C_{1}$, $\rho=C_{2} /\left(L G^{2}\right), r_{0}=R_{0} C_{2} /(L G), a=G_{a} / G b=G_{b} / G$ and $\tau=G t / C_{2}$. The following non-dimensional equations of the system can be formulated,

$$
\left\{\begin{array}{l}
\dot{x}_{1}=\varepsilon\left(x_{2}-(1+b) x_{1}-\frac{a-b}{2}\left(\left|x_{1}+1\right|-\left|x_{1}-1\right|\right)\right), \\
\dot{x}_{2}=x_{1}-x_{2}+x_{3} \\
\dot{x}_{3}=-\rho x_{2}-r_{0} x_{3}-\rho u .
\end{array}\right.
$$

From Fig. 1, we have that the output is $y=v_{1}$. To facilitate the controller design, we re-arrange (38) into the state-space form of (1) with

$$
\begin{aligned}
& A=\left[\begin{array}{ccc}
-\varepsilon(1+b) & \varepsilon & 0 \\
1 & -1 & 1 \\
0 & -\rho & -r_{0}
\end{array}\right], \quad B=-\left[\begin{array}{l}
0 \\
0 \\
\rho
\end{array}\right], \\
& \phi=\left[\begin{array}{c}
0 \\
0 \\
\frac{a-b}{2}\left(\left|x_{1}+1\right|-\left|x_{1}-1\right|\right)
\end{array}\right], C=\left[\begin{array}{lll}
E & 0 & 0
\end{array}\right] \text {. }
\end{aligned}
$$

For the simulation study, the following values of the circuit parameters are chosen:

$C_{1}=10 \mathrm{nF}, C_{2}=100 \mathrm{nF}, L=18.68 \mathrm{mH}, R_{0}=16 \Omega$,

$G_{a}=-0.75 \mathrm{mS}, G_{b}=-0.41 \mathrm{mS}, E=1 \mathrm{~V}, R=1.75 \mathrm{k} \Omega$.

The circuit parameters imply that $\varepsilon=10, \rho=16.3945, r_{0}=$ $0.1499, a=-1.3125$ and $b=-0.7175$. The time delay $h=$ $0.03 \mathrm{~s}$ of the system is fixed, and the Lipschitz constant $\gamma=$ 5.95 is computed.

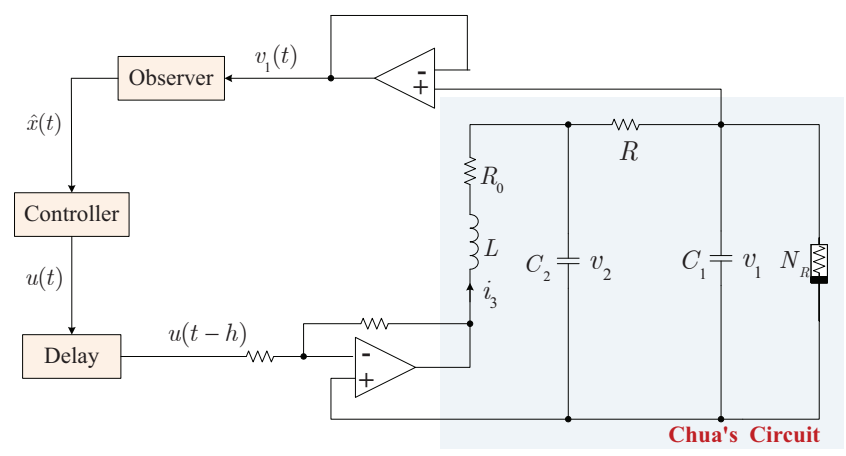

Fig. 1. Output feedback Chua's circuit.

With $\alpha=0.01$ and $\omega_{1}=\omega_{2}=0.1$, the solutions for $P_{1}$ and $P_{2}$ to satisfy (33)-(37) can be calculated as

$$
P_{1}=W^{-1}=\left[\begin{array}{lll}
0.0045 & 0.0121 & 0.0042 \\
0.0121 & 0.0703 & 0.0045 \\
0.0042 & 0.0045 & 0.0064
\end{array}\right],
$$

and

$$
P_{2}=\left[\begin{array}{ccc}
0.5081 & -0.4567 & 0.2043 \\
-0.4567 & 1.4390 & -0.1414 \\
0.2043 & -0.1414 & 0.1573
\end{array}\right] .
$$

By (12), we have

$$
K=[0.0689,0.0738,0.1049],
$$

and

$$
L=\left[\begin{array}{c}
-5.3798 \\
-1.1193 \\
5.9824
\end{array}\right]
$$

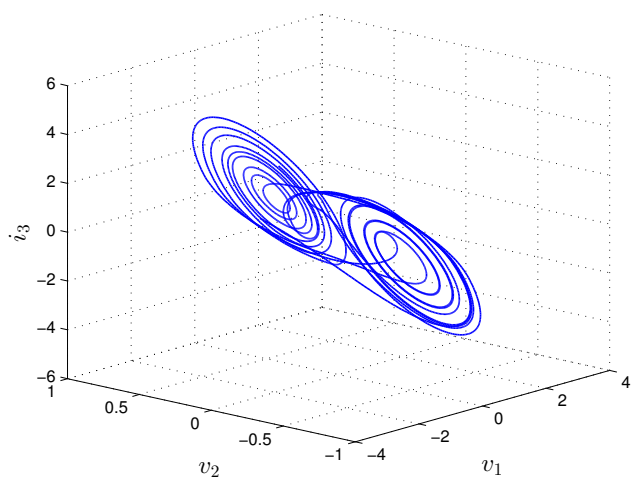

Fig. 2. Open-loop phase portrait. 
The initial conditions $x(0)=[1,0.8,-0.9]^{\mathrm{T}}$ and $\hat{x}(0)=$ $[0.1,0,0]^{\mathrm{T}}$ are chosen for the system (38) and the observer (5), respectively. The open-loop dynamic responses are plotted in Fig. 2, which shows the chaotic "double scroll" attractor pattern. With the inclusion of a voltage feedback loop into the nominal Chua's circuit, the asymptotic stability of the closedloop system in the presence of input delay is achieved, as shown in Figs. 3-5.

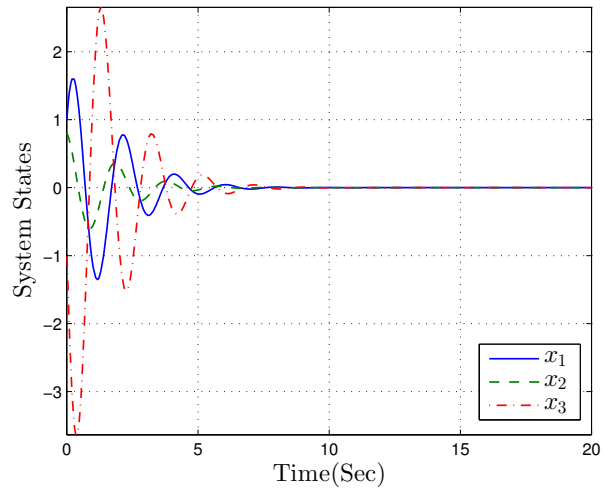

Fig. 3. Closed-loop responses: $h=0.03$.

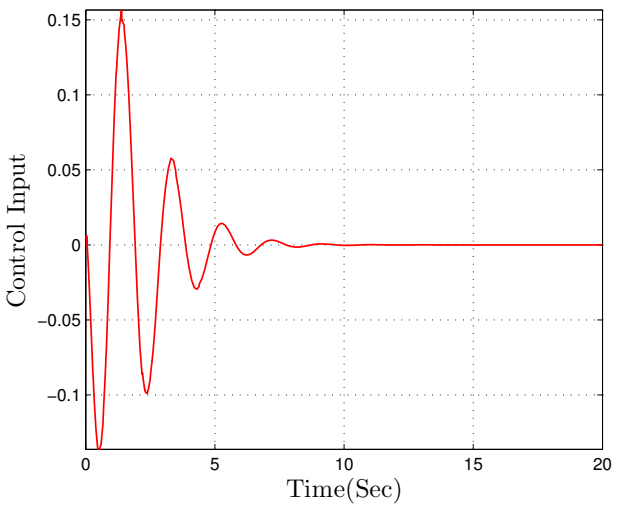

Fig. 4. Control input: $h=0.03$.

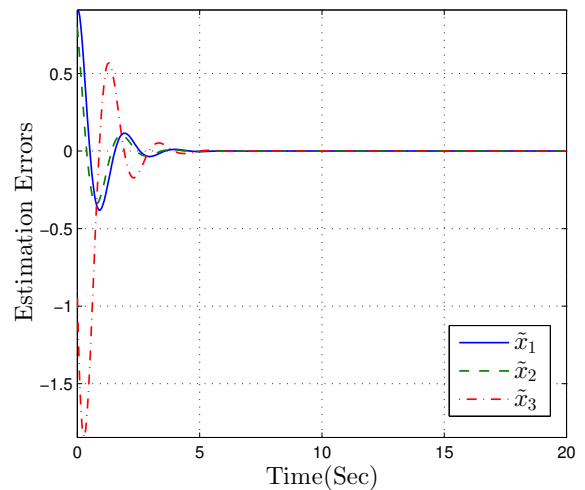

Fig. 5. Estimation errors: $h=0.03$.

\section{Conclusions}

In this brief, we have developed a truncated prediction output feedback design for a class of Lipschitz nonlinear systems with input delay. The stability analysis is carried out in the framework of Lyapunov-Krasovskii functionals. Sufficient conditions for the asymptotic stability are presented in LMIs with a set of iterative scalar parameters. The proposed design method has been successfully applied to stabilize a chaotic circuit with input delay by output feedback.

\section{REFERENCES}

[1] E.R. Samuel, L. Knockaert, and T. Dhaene, "Matrix-interpolation-based parametric model order reduction for multiconductor transmission lines with delays," IEEE Trans. Circuits Syst. II, Exp. Briefs, vol. 62, no. 3, pp. 276-280, 2015.

[2] J. Richard, "Time-delay systems: An overview of some recent advances and open problems," Automatica, vol. 39, no. 10, pp. 1667-1694, 2003.

[3] O. Smith, "A controller to overcome dead time," ISA Journal, vol. 6 no. 2, pp. 28-33, 1959.

[4] A. Manitius and A. Olbrot, "Finite spectrum assignment problem for systems with delays," IEEE Trans. Autom. Control, vol. 24, no. 2, pp. 541-552, 1979.

[5] K. Engelborghs, M. Dambrine, and D. Roose, "Limitations of a class of stabilization methods for delay systems," IEEE Trans. Autom. Control, vol. 46, no. 2, pp. 336-339, 2001

[6] S. Mondie and W. Michiels, "Finite spectrum assignment of unstable time-delay systems with a safe implementation," IEEE Trans. Autom. Control, vol. 48, no. 12, pp. 2207-2212, 2003.

[7] Z. Lin, Low Gain Feedback. London: Springer-Verlag, 1998.

[8] Z. Lin and H. Fang, "On asymptotic stabilizability of linear systems with delayed input," IEEE Trans. Autom. Control, vol. 52, no. 6, pp. 998-1013, 2007.

[9] B. Zhou, Z. Lin, and G. Duan, "Stabilization of linear systems with input delay and saturation - a parametric Lyapunov equation approach," Int. J. Robust. Nonlinear Control, vol. 20, no. 13, pp. 1502-1519, 2010.

[10] B. Zhou, Z. Lin, and G. Duan, "Truncated predictor feedback for linear systems with long time-varying input delays," Automatica, vol. 48, no. 10 , pp. 2387-2399, 2012.

[11] S.Y. Yoon and Z. Lin, "Truncated prediction feedback control for exponentially unstable linear systems with time-varying input delay," Systems \& Control Letters, vol. 62, no. 10, pp. 837-844, 2013.

[12] F. Mazenc and P.-A. Bliman, "Backstepping design for time-delay nonlinear systems," IEEE Trans. Autom. Control, vol. 51, no. 1, pp. 145-149, 2006.

[13] M. Krstic, Delay compensation for nonlinear, adaptive, and PDE systems. Boston, MA: Birkhauser, 2009.

[14] M. Krstic, "Input delay compensation for forward complete and strictfeedforward nonlinear systems," IEEE Trans. Autom. Control, vol. 55, no. 2, pp. 287-303, 2010.

[15] F. Mazenc, S.-I. Niculescu, and M. Krstic, "Lyapunov-Krasovskii functionals and applications to input delay compensation for linear-timeinvariant systems," Automatica, vol. 48, no. 7, pp. 1317-1323, 2012.

[16] W. Pasillas-Lépine, A. Loría, and T. B. Hoang, "Preliminary results on output tracking control for restricted-feedback linearizable systems with input delay," in Proc. 52nd IEEE Conf. Decision Control, Florence, Italy, 1995, pp. 324-329.

[17] Z. Ding and Z. Lin, "Truncated state prediction for control of Lipschitz nonlinear systems with input delay," in Proc. 53rd IEEE Conf. Decision Control, Los Angeles, CA, USA, 2014, pp. 1966-1971

[18] R. Rajamani, "Observers for lipschitz nonlinear systems," IEEE Trans. Autom. Control, vol. 43, no. 3, pp. 397-401, 1998.

[19] C. Aboky, G. Sallet and J. Vivalda, "Observer for lipschitz non-linear systems," Int. J. Control, vol. 75, no. 3, pp. 204-212, 2002.

[20] Z. Ding, Nonlinear and Adaptive Control Systems, London: IET, 2013.

[21] S. Y. Yoon, P. Anantachaisilp, and Z. Lin, "An LMI approach to the control of exponentially unstable systems with input time delay," in Proc. 52nd IEEE Conf. Decision Control, Florence, Italy, 2013, pp. 312-317.

[22] K. Gu, "An integral inequality in the stability problem of time-delay systems," in Proc. 39th IEEE Conf. Decision Control, Sydney, Australia, 2010 , pp. 2805-2810.

[23] X. Wang, G. Zhong, K. Tang, K. Man and Z. Liu, "Generating chaos in Chua's circuit via time-delay feedback," IEEE Trans. Circuits Syst. I, Reg. Papers, vol. 48, no. 9, pp. 1151-1156, 2001. 